\title{
Inhibition of pregnane $X$ receptor pathway contributes to the cell growth inhibition and apoptosis of anticancer agents in ovarian cancer cells
}

\author{
HISASHI MASUYAMA, KEIICHIRO NAKAMURA, ETSUKO NOBUMOTO and YUJI HIRAMATSU \\ Department of Obstetrics and Gynecology, Okayama University Graduate School of Medicine, \\ Dentist and Pharmaceutical Science, Okayama, Japan
}

Received May 9, 2016; Accepted June 28, 2016

DOI: $10.3892 /$ ijo.2016.3611

\begin{abstract}
Epithelial ovarian cancer remains the most devastating gynecologic cancer with drug resistance and rapid recurrence. Pregnane $\mathrm{X}$ receptor (PXR) is a nuclear receptor that affects drug metabolism/efflux and drug-drug interaction through control of multiple drug resistance 1 (MDRl), which implies a major role in multidrug resistance, and other genes. We examined whether the inhibition of PXR-mediated pathway using siRNA interference and an antagonist for PXR could influence the paclitaxel and cisplatin cytotoxicity in ovarian cancer cells. PXR agonists, phthalate and pregnenolone had significant positive effects on cytochrome P450 (CYP) 3A4 expression and PXR-mediated transcription through the CYP3A4 promoter, whereas MDR1 expression and PXR-mediated transcription though the $M D R 1$ promoter were significantly increased in the presence of paclitaxel or cisplatin. Downregulation of PXR suppressed the augmented MDR1 expression and PXR-mediated transcription by PXR ligands, and significantly enhanced cell growth inhibition and apoptosis in the presence of paclitaxel or cisplatin. Additionally, ketoconazole, a PXR antagonist, suppressed the augmented MDR1 expression and PXR-mediated transactivation by paclitaxel and cisplatin, and enhanced cell growth inhibition and apoptosis in their presence. In conclusion, inhibition of PXR-mediated pathways could be a novel means of augmenting sensitivity, or overcoming resistance to anticancer agents for ovarian cancer.
\end{abstract}

\section{Introduction}

Epithelial ovarian cancer remains the most devastating gynecologic cancer. Although 40-60\% of patients achieve complete

Correspondence to: Dr Hisashi Masuyama, Department of Obstetrics and Gynecology, Okayama University Graduate School of Medicine, Dentist and Pharmaceutical Science, 2-5-1 Shikata, Okayama 700-8558, Japan

E-mail: masuyama@cc.okayama-u.ac.jp

Key words: ovarian cancer, pregnane $\mathrm{X}$ receptor, paclitaxel, cisplatin, multiple drug resistance 1 clinical responses to first-line chemotherapy treatment, $\sim 50 \%$ of these patients relapse within 5 years; only $10-15 \%$ of patients who present with advanced disease achieve long-term remission $(1,2)$. The standard of care for first-line chemotherapy treatment is paclitaxel in combination with a platinum-based compound, or platinum-based therapy alone (3). Chemotherapy induces significant side effects and has limited efficacy, such as drug resistance and rapid recurrence, respectively, which subsequently reduce patient survival rates $(4,5)$. Hence, more effort is needed to understand the mechanisms of drug resistance to anticancer drugs. Resistance to chemotherapy for ovarian cancer potentially involves several mechanisms (6). P-glycoprotein, which is encoded by the gene, multiple drug resistance $1(M D R 1)$, functions as a transmembrane efflux pump for the elimination and disposition of xenobiotics, including the anticancer drugs paclitaxel and cisplatin, which implies a major role in multidrug resistance (7). MDR1 overexpression in certain tumor cells has been associated with protection against anticancer agents (8). Therefore, altering $M D R 1$ expression could improve the patient's clinical outcome (9-11).

Pregnane $\mathrm{X}$ receptor (PXR), a member of the nuclear receptor superfamily, has been shown to mediate the genomic effects of several steroid hormones, including progesterone, pregnenolone, and estrogen, and of xenobiotics (12-18). PXR regulation involves a specific DNA sequence, the PXR-responsive element, which is found in various genes, including the upstream region of the cytochrome (P450) 3A (CYP3A) gene family $(12,14,17)$, which codes for monooxygenases responsible for the oxidative metabolism of certain endogenous substrates and xenobiotics $(19,20)$, and MDR1 $(21,22)$. Because the PXR pathway is activated by a large number of prescription drugs designed to treat infection, cancer, convulsion, and hypertension (23), PXR is thought to play roles in drug metabolism/efflux and drug-drug interaction. For example, PXR activation is reportedly involved in regulating cell cycle proliferation and apoptosis inhibition (24-27). PXR was previously shown as a possible prognostic factor that might feasibly identify patients at risk of recurrence or death from epithelial ovarian cancer (28), however, PXR function in ovarian cancer remains unknown, especially in combination with anticancer drugs. Paclitaxel and cisplatin, 
which are widely used as first-line chemotherapy drugs in ovarian cancer (3-5), could act as PXR ligands (29).

In addition, we previously showed that downregulating the constitutive androstane receptor (CAR) through RNA interference significantly promoted cell growth inhibition and enhancement of apoptosis in the presence of anticancer agents, paclitaxel and cisplatin (30). CAR as well as PXR is activated by a variety of endogenous and exogenous ligands including drugs, insecticides, pesticides, and nutritional compounds (31) and functions as a xenobiotic receptor that regulates detoxification and clearance of xenobiotics (32). Therefore, both PXR and CAR might play some roles in drug resistance.

We therefore examined the effects of combining a ligand and antagonist for PXR with anticancer drugs on ovarian cancer cells, and the potential contribution of PXR downregulation by RNA interference toward increasing drug sensitivity and overcoming drug resistance in this study. We also examined the relationship of PXR with another xenobiotics receptor $\mathrm{CAR}$ in the drug resistance function for ovarian cancer.

\section{Materials and methods}

Materials. Phthalic acid bis (2-ethylhexel ester) (phthalate), 5-pregneno-3 $\beta$-ol-20-one (pregnenolone), ketoconazole, paclitaxel, cisplatin, and dimethyl sulfoxide (DMSO) were purchased from Sigma-Aldrich (St. Louis, MO, USA). Each drug was dissolved in DMSO and stored at $-20^{\circ} \mathrm{C}$ before use. The final concentration of DMSO was $0.1 \%$; $0.1 \%$ DMSO was used for the negative control.

Cell culture. Ovarian cancer cell lines, SKOV-3, OVCAR-3, CaOV-3, and BG-1 cells, and human hepatoma cell line, HepG2 cells were obtained from the Health Science Research Resources Bank (Osaka, Japan). These ovarian cancer cell lines were derived from patients with ovarian adenocarcinoma. SKOV-3 cells and CAOV-3 were from patients previously treated with chemotherapy, BG-1 cells from an untreated patient, and OVCAR-3 cell line from a patient who did not respond to chemotherapy (33). SKOV-3 and OVCAR-3 cells have been shown to be relatively resistant to platinum and taxanes, BG-1 cells to be resistant to platinum, and CAOV-3 to be sensitive to both platinum and taxanes $(33,34)$. Cells were cultured in Dulbecco's modified Eagle's medium (Invitrogen, Carlsbad, CA, USA) with $10 \%$ charcoal-stripped fetal bovine serum (Biological Industries, Kibbutz Beit Haemek, Israel) and $1 \%$ penicillin/streptomycin (Invitrogen). The cells were cultured under a humidified $5 \% \mathrm{CO}_{2}$ atmosphere at $37^{\circ} \mathrm{C}$.

Quantitative real-time reverse-transcription polymerase chain reaction $(P C R)$. Total RNA was isolated using the RNeasy Mini kit (Qiagen, Hilden, Germany) according to the manufacturer's protocol, then stored at $-80^{\circ} \mathrm{C}$. The concentration and purity of isolated RNA was determined using a spectrophotometer (Thermo Fisher Scientific Inc. Waltham, MA, USA). Reverse transcription was performed using the High Capacity cDNA Reverse Transcription kit with RNA inhibitor (Applied Biosystems, Foster City, CA, USA). Complementary DNA was obtained through the GeneAmp PCR System 9700 (Applied Biosystems). cDNA isolated from each of the different drug groups was analyzed by real-time
PCR using TaqMan universal master mix containing different primers (Applied Biosystems). Primers for MDR1, CYP3A4, and PXR (Applied Biosystems) were normalized to the $18 \mathrm{~S}$ housekeeping gene. After 50 cycles, mRNA expression was calculated by the comparative CT-value method. The negative control was $0.1 \%$ DMSO.

Transient transfection studies. The (CYP3A4) ${ }^{3}$-tk-chloramphenicol acetyl transferase (CAT) was generated by inserting three copies of double-strand oligonucleotides containing the CYP3A4 (5'-GGGTCAGCAAGTTCA-3'). The (MDR1) ${ }^{3}$ tk-CAT was generated by inserting three copies of a double-strand oligonucleotide containing the MDR1 (5'-AGGTCAAGTTA GTTCA-3') as described before (29). CaOV-3 cells were transfected with $1 \mu$ g of a reporter gene construct [(CYP3A4) $)^{3}$-tk-CAT or (MDR1) ${ }^{3}$-tk-CAT]. In all experiments, liposome-mediated transfections used Lipofectamine (Life Technologies, Gaithersburg, MD, USA) according to the manufacturer's instructions. Transfected cells were treated with DMSO alone or with anticancer drugs paclitaxel $(5 \mu \mathrm{M})$, cisplatin $(10 \mu \mathrm{M})$, PXR ligands pregnenolone $(10 \mu \mathrm{M})$ or phthalate $(10 \mu \mathrm{M})$, with or without RNA interference for $36 \mathrm{~h}$. Cell extracts were prepared and assayed for CAT activity. Amount of CAT was determined using a CAT ELISA Kit (Roche Diagnostics Co., Tokyo, Japan) according to the manufacturer's instructions.

RNA interference. The siRNA cocktail targeting human PXR (cat. no. sc-44057), human CAR (cat. no. sc-39918), and negative control cocktail (cat. no. sc-37007), which consists of a scrambled sequence that does not lead to the specific degradation of any cellular message, were purchased from Santa Cruz Biotechnology (Santa Cruz, CA, USA). Cells were transfected with PXR siRNA, CAR siRNA or control siRNA using the siRNA Reagent system (Santa Cruz Biotechnology) according to the manufacturer's protocol.

MTT assay. Cells were plated $5 \times 10^{3}$ in a 96 -well plate in at least triplicate samples for each experimental condition. Twenty-four hours later, cells were treated with anticancer drugs paclitaxel $(5 \mu \mathrm{M})$ or cisplatin $(10 \mu \mathrm{M})$ with or without RNA interference or in the presence or absence of pregnenolone or ketoconazole for 24, 48 and $72 \mathrm{~h}$. We used a commercial MTT cell proliferation kit (Bioassay Systems, Hayward, CA, USA) and followed its instructions. After each incubation time, $15 \mu 1$ 3-(4,5-dimethylthiazol-2-yl)-2,5-diphenyltetrazolium bromide (MTT) was added into each well. After a 4-h incubation at $37^{\circ} \mathrm{C}, 150 \mu \mathrm{l}$ solubilization solution was added to each well and the plate was gently agitated for $1 \mathrm{~h}$ at room temperature. Absorbance was measured at $595 \mathrm{~nm}$ using an Imark microplate reader (Bio-Rad, Philadelphia, PA, USA).

Apoptosis assay. Apoptosis was examined using a terminal deoxynucleotidyl transferase-mediated dUTP nick-end labeling (TUNEL) assay that employed the ApopTag Plus Peroxidase In Situ Apoptosis Detection kit (EMD Millipore, Billerica, MA, USA) following the manufacturer's protocol. Briefly, cells were grown on sterilized glass coverslips in a 6-well culture plate overnight, and then exposed to the different experimental drug groups. Cells were then stained and mounted with Vectashield mounting medium in the 
A

\begin{tabular}{|c|c|c|c|c|}
\hline & SKOV-3 & BG-1 & OVCAR-3 & CaOV-3 \\
\hline PXR & & & 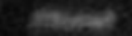 & 20 \\
\hline
\end{tabular}

B

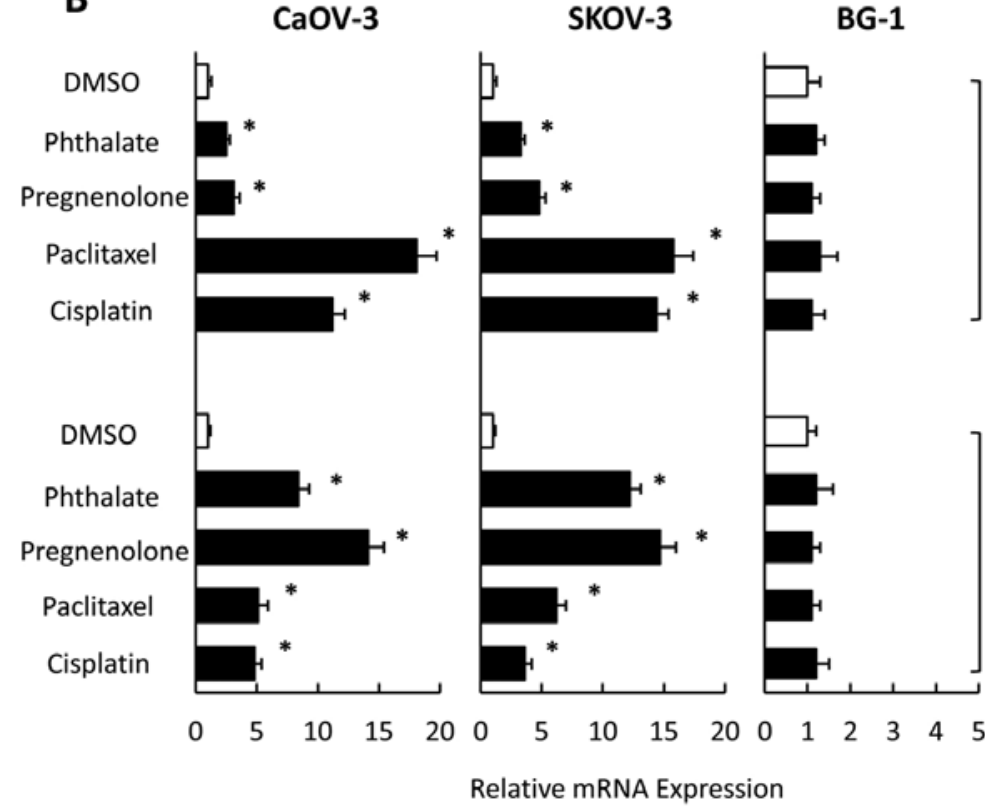

C

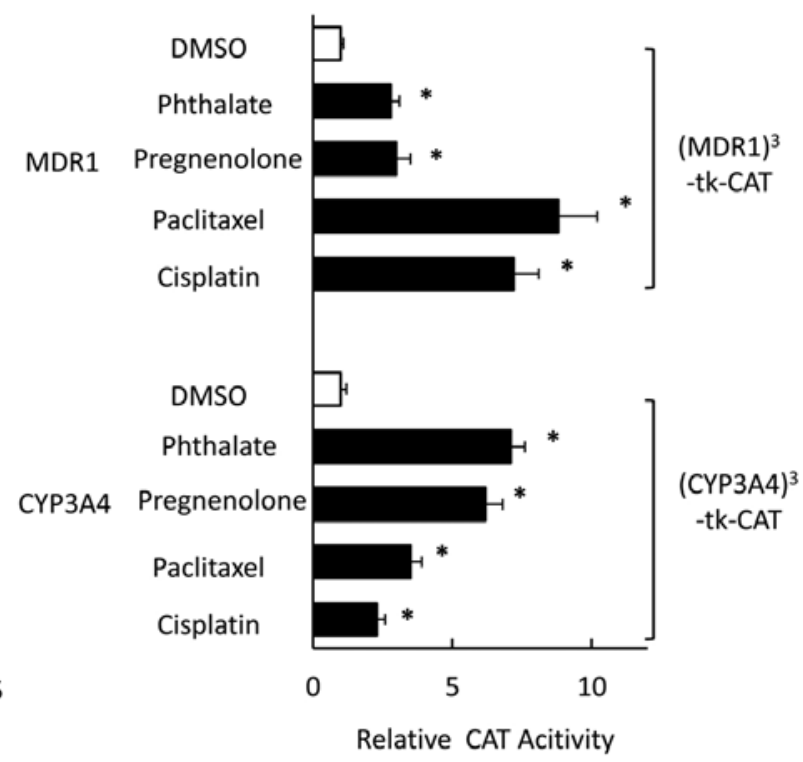

Figure 1. Effect of anticancer drugs on the mRNA expression of MDR1 and CYP3A4 and PXR-mediated transcription in ovarian cancer cell lines. The expression of PXR and GAPDH mRNAs were analyzed using RT-PCR in ovarian cancer cell lines, SKOV-3, BG-1, OVCAR-3, and CaOV-3 (A). CaOV-3, SKOV-3, and BG-1 cells were treated with pregnenolone, phthalate, paclitaxel, cisplatin, or DMSO for $24 \mathrm{~h}$ and analyzed for the mRNA expression using quantitative real-time PCR (B). CaOV-3 cells were cotransfected with reporter gene constructs, (MDR1) ${ }^{3}$-tk-CAT or (CYP3A4) ${ }^{3}$-tk-CAT and treated with pregnenolone, phthalate, paclitaxel, cisplatin, or DMSO for $36 \mathrm{~h}$. CAT levels were determined by an ELISA kit (C). Results show mean \pm SD of 5 independent experiments ( $\mathrm{P}<0.01$ vs. DMSO group).

presence of the nuclear stain 4',6-diamidino-2-phenylindole (DAPI). Afterwards, TUNEL- and DAPI-positive cells were counted using fluorescence microscopy. The ratio of TUNELpositive to DAPI-positive cells was calculated.

Statistical analysis. Statistical analysis was performed as one-way analysis of variance with post-hoc testing analysis and the Student's t-test using SPSS Statistics 19.0 software (IBM, Armonk, NY, USA). All data are expressed as mean $\pm \mathrm{SD}$. $\mathrm{P}<0.05$ was considered statistically significant.

\section{Results}

Effect of paclitaxel and cisplatin on MDR1 and CYP3A4 $m R N A$ expression and PXR-mediated transcription in ovarian cancer cell lines. Expression of PXR has been reported in SKOV-3 and OVCAR-8 ovarian cancer cells (27). We examined PXR expression in OVCAR-3, CaOV-3, SKOV-3 and BG-1 cells using RT-PCR. PXR was strongly expressed in SKOV-3 and CaOV-3 cells, moderately expressed in OVCAR-3 cells, and not expressed in BG-1 cells (Fig. 1A). To investigate the effect of PXR ligands (including anticancer drugs) on expression of CYP3A4 and MDR1 in vitro, we examined mRNA levels of CYP3A4 and MDR1 in SKOV-3, CaOV-3, and
BG-1 cells that had been exposed to pregnenolone, phthalate, paclitaxel, and cisplatin, which could activate PXR-mediated transcription through CYP3A4 and MDR1 promoters (28). In SKOV-3 and CaOV-3 cells, CYP3A4 mRNA levels were significantly increased in the presence of PXR ligands (Fig. 1B). Pregnenolone and phthalate had significantly positive effects on CYP3A4 expression compared with paclitaxel or cisplatin. In contrast, the MDR1 level was significantly and strongly increased in the presence of paclitaxel or cisplatin compared with pregnenolone and phthalate. MDR1 and CYP3A4 levels did not change in response to any PXR ligands in BG-1 cells (which did not express PXR). PXR ligands also enhanced PXR-mediated transcription through both MDR and CYP3A4 promoters in CaOV-3 cells (Fig. 1C). Paclitaxel and cisplatin had significant effects on PXR-mediated transcription through the MDR promoter compared with pregnenolone and phthalate. In contrast, pregnenolone and phthalate strongly enhanced PXR-mediated transcription through CYP3A4 promoter compared with paclitaxel or cisplatin.

Effect of PXR siRNA on MDRl and CYP3A4 mRNA expression and PXR-mediated transcription in CaOV-3 cells. We examined the effect of downregulating PXR by using siRNA on expression of MDR1 and CYP3A4, and PXR-mediated 

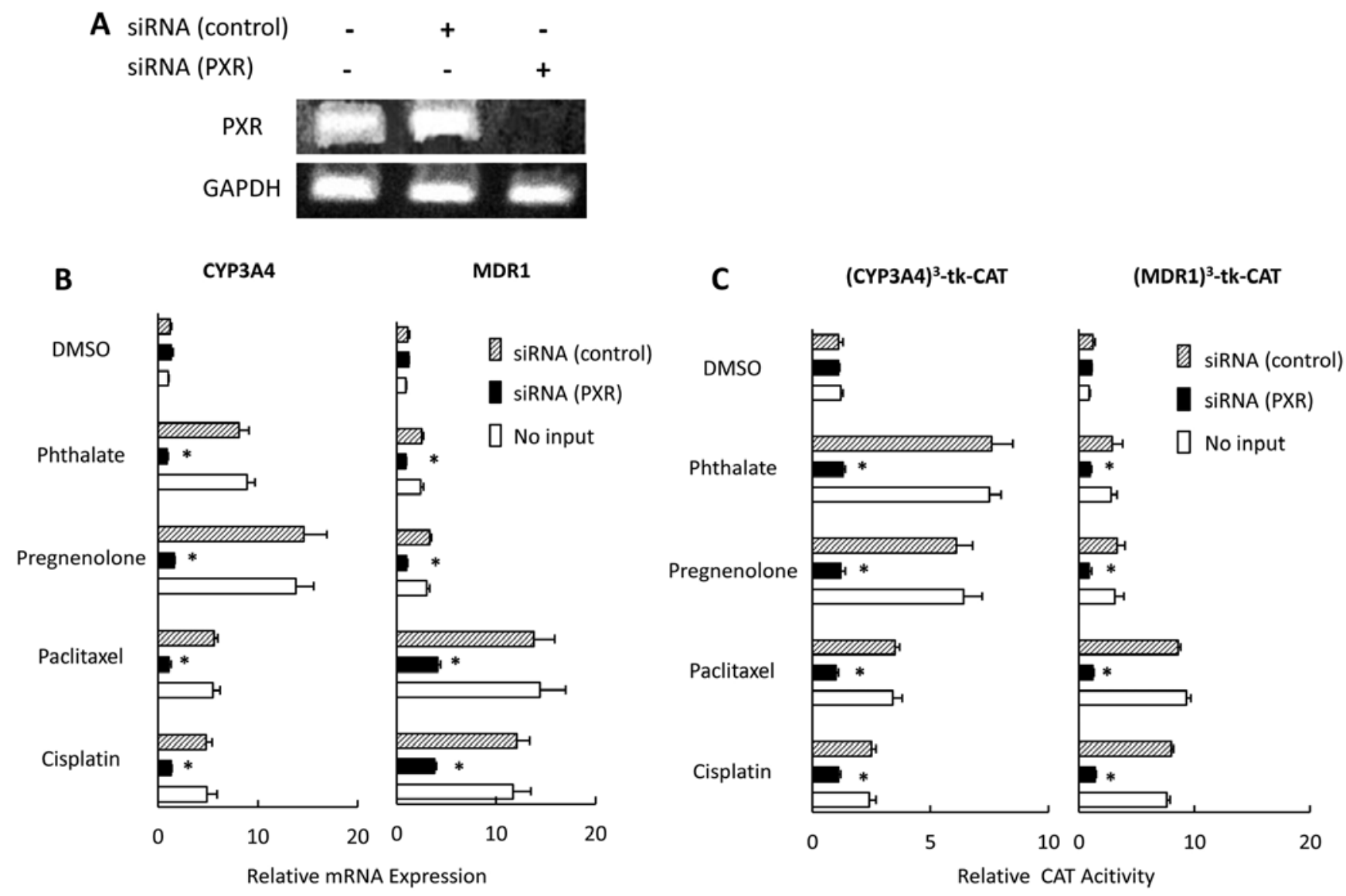

Figure 2. Effect of PXR siRNA on the expression of MDR1 and CYP3A4 and PXR-mediated transcription in CaOV-3 cells. CaOV-3 cells were transfected with PXR- or control siRNA (A). CaOV-3 cells were transfected with PXR- or control siRNA and treated with pregnenolone, phthalate, paclitaxel, cisplatin, or DMSO for $36 \mathrm{~h}$. CYP3A4 and MDR1 mRNA expression were analyzed by quantitative real-time PCR. Each experiment was performed in triplicate, 4 times (internal control, 18S; negative control, DMSO). Changes in gene expression were calculated as ratios of target gene to internal control (B). CaOV-3 cells were cotransfected with PXR- or control siRNAs, or no siRNA and a reporter gene construct, (MDR1) ${ }^{3}$-tk-CAT or (CYP3A4) ${ }^{3}$-tk-CAT; and then treated with pregnenolone, phthalate, paclitaxel, cisplatin or DMSO for $36 \mathrm{~h}$. CAT levels were determined by ELISA (C). Results, mean \pm SD of 5 independent experiments ( $\mathrm{P}<0.01$ vs. control siRNA group).

transcription in the presence of paclitaxel or cisplatin in CaOV-3 cells. First, we used RT-PCR to confirm that PXR mRNA was not detected in the PXR-siRNA transfected CaOV-3 cells (Fig. 2A). The effects of PXR ligands, including paclitaxel and cisplatin, on MDR1 expression were strongly inhibited in PXR siRNA-transfected cells, and we saw no positive effects of PXR ligands on CYP3A4 expression in PXR siRNA-transfected cells (Fig. 2B), nor any non-specific effects of siRNA. PXR expression did not significantly differ between cells treated with control siRNA and those not treated with any siRNA (data not shown). We also examined the effects of PXR siRNAs on PXR-mediated transcription. In control siRNA-transfected cells, PXR ligands significantly activated native PXR-mediated transcription; whereas the PXR siRNAtransfected cells showed no PXR-mediated transactivation in the presence of PXR ligands, including paclitaxel and cisplatin (Fig. 2C).

Effect of PXR siRNA on cell proliferation and apoptosis in $\mathrm{CaOV}-3$ cells. We also examined the effect of downregulated PXR on cell proliferation and apoptosis in the presence of paclitaxel or cisplatin in CaOV-3 cells. We found that downregulated PXR significantly enhanced cell growth inhibition in the presence of paclitaxel (Fig. 3A) and cisplatin (Fig. 3B) for 24,48 , and $72 \mathrm{~h}$. Cell growth did not significantly differ between control siRNA-transfected cells and untransfected cells. We also observed that downregulated PXR significantly enhanced apoptosis in PXR siRNA-transfected cells compared with cells transfected with control siRNA or without siRNA in the presence of paclitaxel (Fig. 3C) and cisplatin (Fig. 3D) for 48 and $72 \mathrm{~h}$. Apoptosis rates did not significantly differ between control siRNA-transfected cells and untransfected cells.

Effect of combining paclitaxel or cisplatin with PXR ligand or antagonist on transcription of PXR-related genes. To determine the effect of treating CaOV-3 cells with anticancer drugs in combination with the PXR agonist pregnenolone, or the PXR antagonist ketoconazole on the PXR target genes, MDR1 and CYP3A4, we used real-time PCR to assess their mRNA expression levels at $24 \mathrm{~h}$. We observed pregnenolone moderately suppressed the MDR1 mRNA expression enhanced by paclitaxel or cisplatin, whereas ketoconazole had a significant negative effect on the MDR1 expression enhanced by paclitaxel (Fig. 4A) or cisplatin (Fig. 4B) in a dose-dependent manner. We also examined the effect on PXR-mediated transcription 

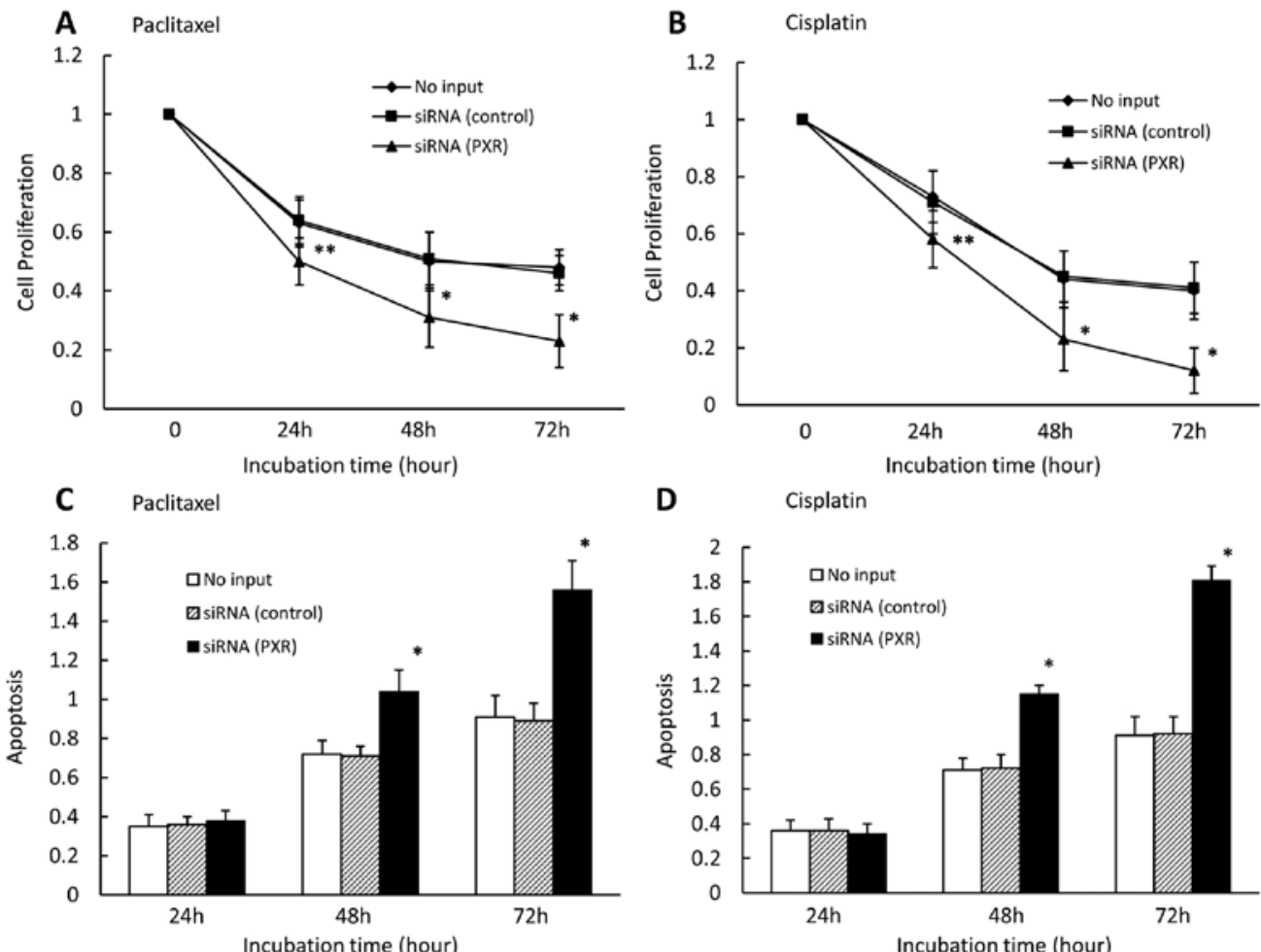

Figure 3. Effects of $P X R$ siRNA on cell proliferation and apoptosis in CaOV-3 cells. CaOV-3 cells were transfected with $P X R$ siRNA, control siRNA, or no siRNA, and were seeded and incubated with paclitaxel (A), or cisplatin (B) for 0, 24, 48 or $72 \mathrm{~h}$. Cell proliferation was then measured by a commercial MTS assay. Apoptosis of CaOV-3 cells transfected with PXR siRNA, control siRNA, or no siRNA and treated with paclitaxel (C) or cisplatin (D) for $36 \mathrm{~h}$ was assayed by TUNEL. Results show mean \pm SD of 5 independent experiments $\left({ }^{*} \mathrm{P}<0.01 ;{ }^{* *} \mathrm{P}<0.05\right.$ vs. control siRNA group).
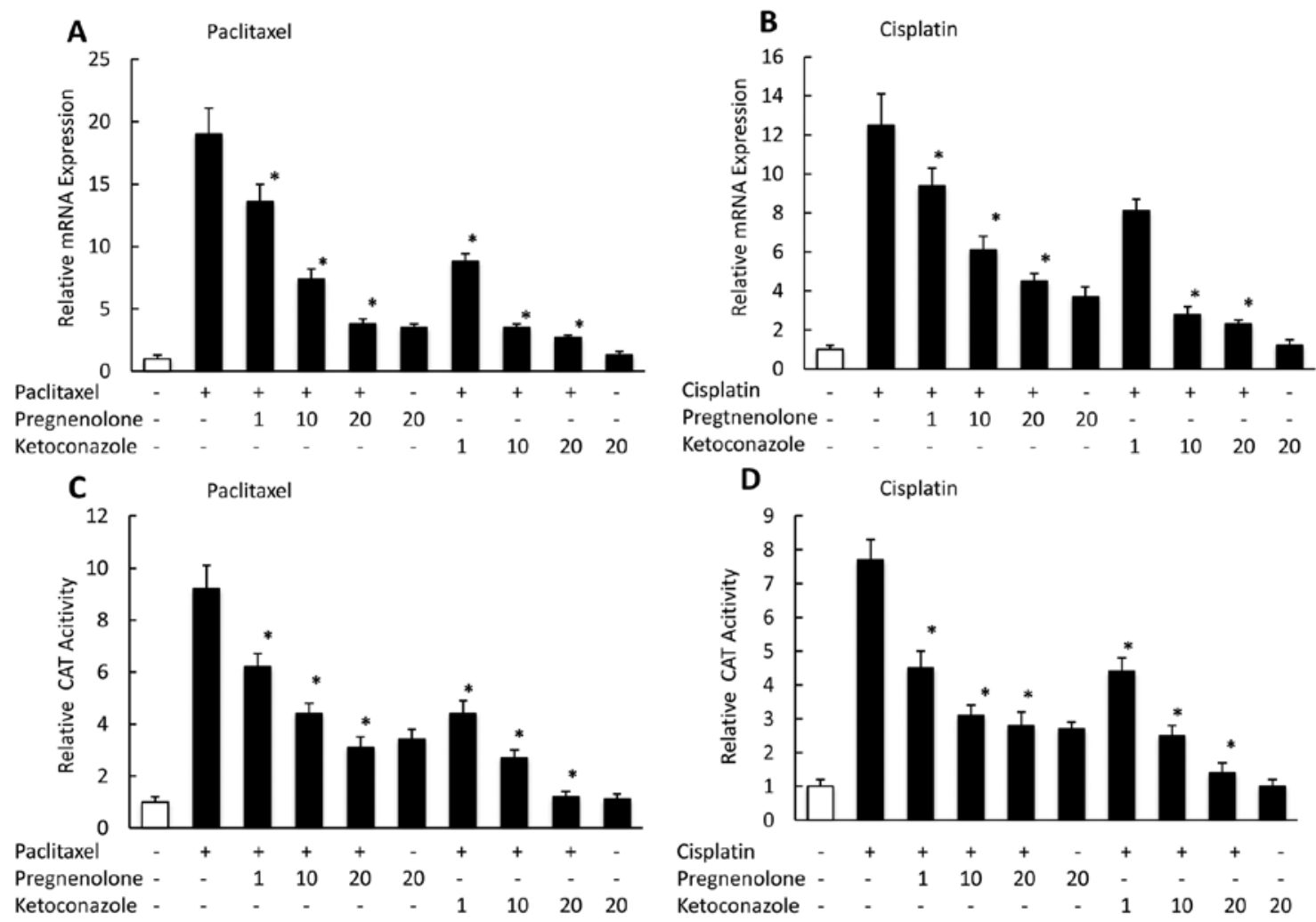

Figure 4. Effect of combining anticancer drugs with PXR ligand or antagonist on the transcription of PXR-related genes. SKOV-3 cells were treated with paclitaxel (A) or cisplatin (B) in the presence or absence of rifampicin or ketoconazole at indicated concentrations for $48 \mathrm{~h}$. MDR1 mRNA expression was analyzed using quantitative real-time PCR. Each experiment was performed in triplicate 4 times (internal control, 18S; negative control, DMSO). Changes in gene expression were calculated as ratio of target gene to internal control. CaOV-3 cells were cotransfected with a reporter gene construct, (MDR1) ${ }^{3}$-tk-CAT, and then treated with paclitaxel (C) or cisplatin (D) in the presence or absence of pregnenolone or ketoconazole at indicated concentrations for $36 \mathrm{~h}$. CAT levels were determined by ELISA. Results show mean \pm SD of 5 independent experiments ( $\mathrm{P}<0.01$ vs. paclitaxel-only). 

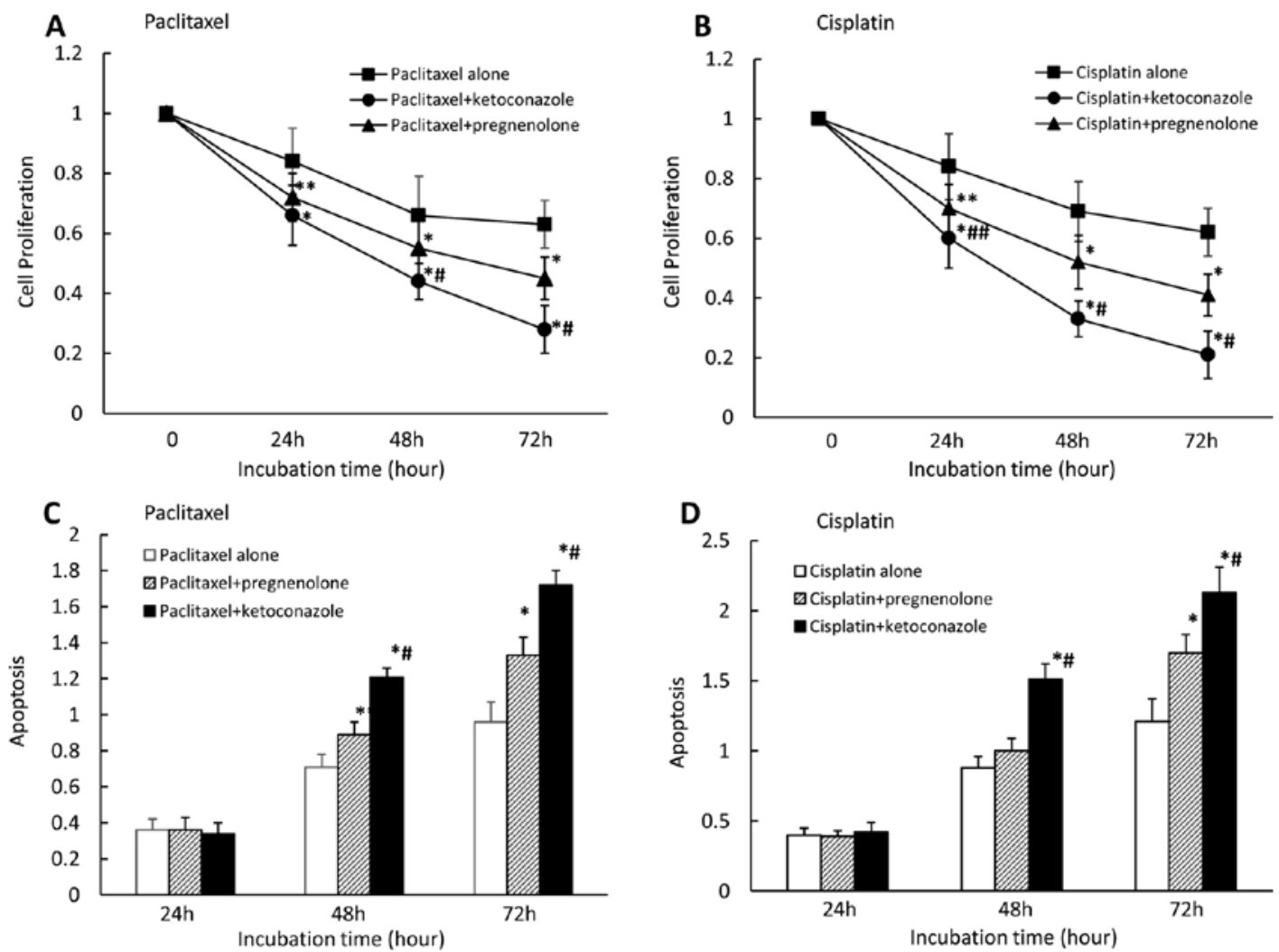

Figure 5. Effect of combining anticancer drugs with PXR ligand or antagonist on cell proliferation and apoptosis in CaOV-3 cells. CaOV-3 cells were transfected with paclitaxel (A) or cisplatin (B) in the presence or absence of pregnenolone or ketoconazole for $0,24,48$ or $72 \mathrm{~h}$. Cell proliferation was then measured with a commercial MTS assay. Apoptosis of CaOV-3 cells, were treated with paclitaxel (C) or cisplatin (D) in the presence or absence of pregnenolone or ketoconazole for 24,48 or $72 \mathrm{~h}$ was assayed by TUNEL. Results show mean $\pm \mathrm{SD}$ of 5 independent experiments $\left({ }^{*} \mathrm{P}<0.01 ;{ }^{* *} \mathrm{P}<0.05\right.$ vs. paclitaxel-only, $\mathrm{P}<0.01$; ${ }^{\# \#} \mathrm{P}<0.05$ vs. rifampicin).

of combining paclitaxel or cisplatin with a PXR ligand or antagonist. Pregnenolone suppressed PXR-mediated transactivation by paclitaxel or cisplatin, whereas ketoconazole more strongly reduced transcription in the presence of paclitaxel (Fig. 4C) and cisplatin (Fig. 4D) in a dose-dependent manner.

Effect of combining paclitaxel or cisplatin with PXR ligand or antagonist on cell proliferation and apoptosis in CaOV-3 cells. We also examined the effects of combining the anticancer paclitaxel or cisplatin with the PXR ligand pregnenolone, or the PXR antagonist ketoconazole on cell proliferation and apoptosis. We found that pregnenolone (moderately) and ketoconazole (strongly) enhanced cell growth inhibition in the presence of paclitaxel (Fig. 5A) or cisplatin (Fig. 5B) after 24,48 and $72 \mathrm{~h}$. Cell growth inhibition significantly differed between pregnenolone and ketoconazole in the presence of paclitaxel for 48 and $72 \mathrm{~h}$ and cisplatin for 24,48 and $72 \mathrm{~h}$. We also observed that pregnenolone (moderately) and ketoconazole (strongly) enhanced apoptosis in the presence of paclitaxel (Fig. 5C) or cisplatin (Fig. 5D) after 48 and $72 \mathrm{~h}$ for either agent. Apoptosis rates significantly differed between pregnenolone and ketoconazole in the presence of either paclitaxel or cisplatin for 48 and $72 \mathrm{~h}$ for either agent.

Effect of PXR siRNA and PXR ligand or antagonist on cell proliferation in other ovarian cell lines treated with paclitaxel or cisplatin. We also examined the effect of PXR downregulation on cell proliferation in other ovarian cancer cell lines. We observed that PXR downregulation significantly enhanced cell growth inhibition in the presence of paclitaxel in SKOV-3 cells, which strongly express PXR (Fig. 6A) but not in BG-1 cells which do not express PXR (Fig. 6B). Cell growth inhibition did not differ between control siRNAtreated and untransfected cells. In examination of the effect on cell proliferation of combining, anticancer drugs with pregnenolone or ketoconazole, pregnenolone (moderately) and ketoconazole (more strongly) enhanced cell growth inhibition in the presence of paclitaxel in SKOV-3 cells (Fig. 6C), but not in BG-1 cells (Fig. 6D). Cell growth inhibition in the presence of paclitaxel for 48 and $72 \mathrm{~h}$ significantly differed between pregnenolone- and ketoconazole-treated SKOV-3 cells.

Effect of PXR and CAR siRNA on MDR1 expression, cell proliferation, and apoptosis in CaOV-3 cells. Finally, we examined the effect of downregulating PXR and CAR on MDR1 expression, cell proliferation, and apoptosis in the presence of paclitaxel in CaOV-3 cells. We observed that the downregulation of both PXR and CAR expression completely blocked the MDR1 expression enhanced by paclitaxel; PXR or CAR inhibited MDR1 expression significantly but not completely (Fig. 7A). MDR1 expression significantly differed between cells with downregulated PXR and those with downregulated CAR in the presence of paclitaxel or cisplatin. Use of siRNA to interfere with both PXR and CAR significantly 

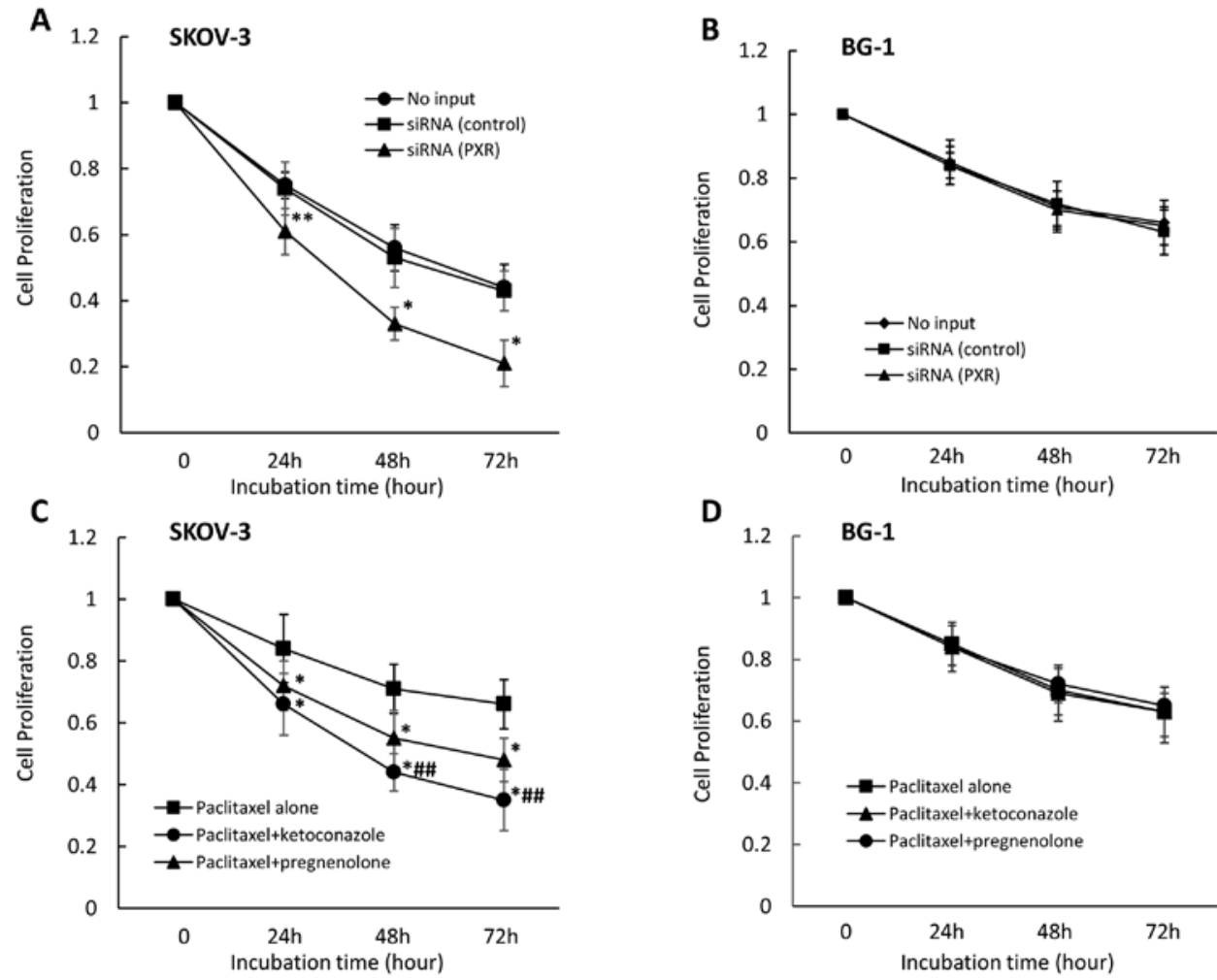

Figure 6. Effects of $P X R$ siRNA and PXR ligand or antagonist on cell proliferation in other ovarian cell lines treated with anticancer drugs. SKOV-3 (A) and BG-1 (B) cells were transfected with PXR siRNA, control siRNA, or no siRNA and were seeded and incubated with paclitaxel for 0, 24, 48 or 72 h. SKOV-3 (C) and BG-1 (D) cells were also treated with paclitaxel in the presence or absence of pregnenolone or ketoconazole for $0,24,48$ or $72 \mathrm{~h}$. Cell proliferation was measured using a commercial MTS assay. Results show mean $\pm \mathrm{SD}$ of 5 independent experiments $\left({ }^{*} \mathrm{P}<0.01 ;{ }^{* *} \mathrm{P}<0.05\right.$ vs. control siRNA or paclitaxel-alone, ${ }^{\# \#} \mathrm{P}<0.05$ vs. paclitaxel+pregnenolone).

A

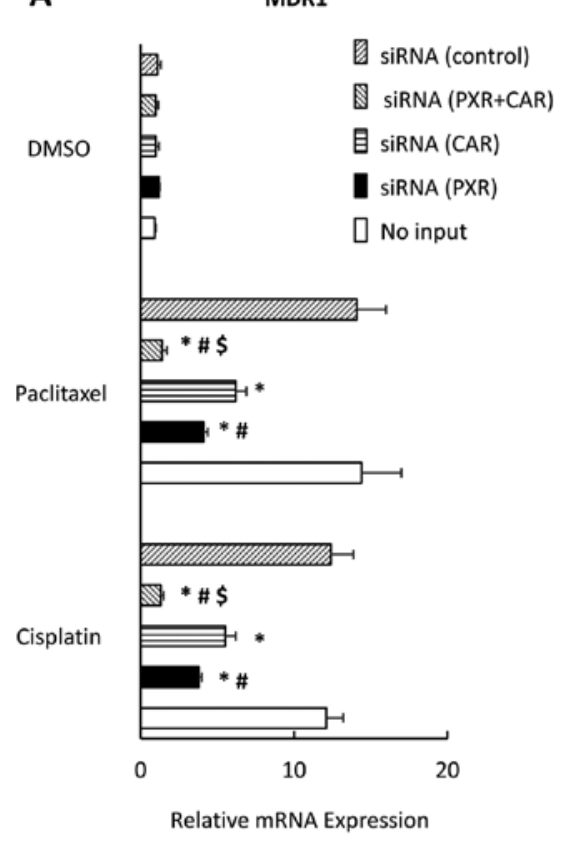

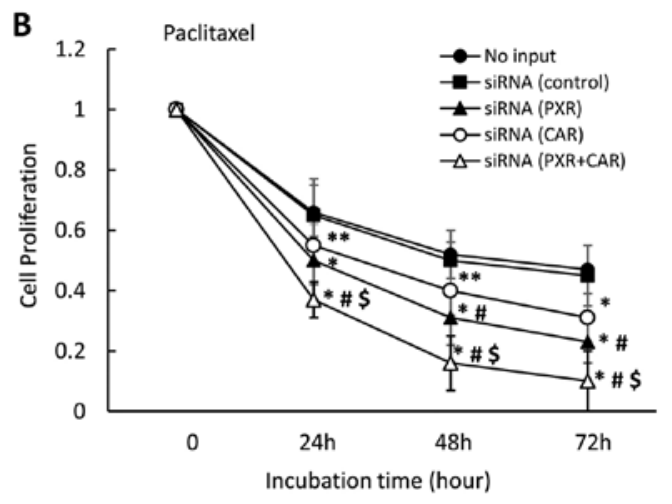

C

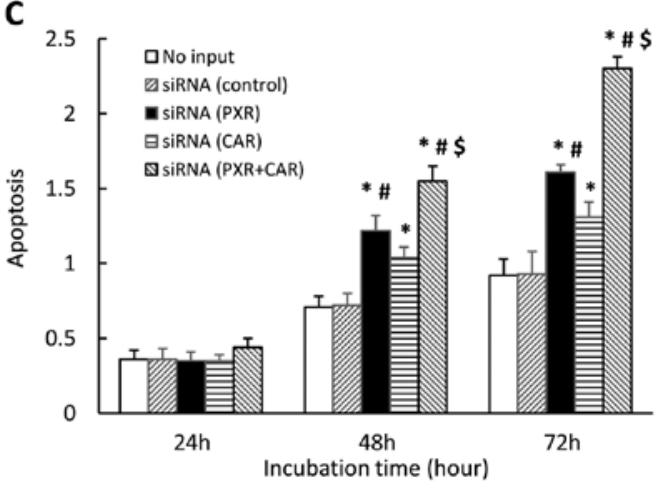

Figure 7. Effect of $P X R$ - and $C A R$ siRNA on MDR1 expression, cell proliferation and apoptosis in CaOV-3 cells. CaOV-3 cells were transfected with $P X R$, $C A R$ or control siRNA and treated with paclitaxel or DMSO for $36 \mathrm{~h}$. MDR1 mRNA expression was analyzed by quantitative real-time PCR. Each experiment was performed in triplicate 4 times (internal control, 18S; negative control, DMSO). Change in gene expression was calculated as ratio of target gene to internal control (A). CaOV-3 cells were transfected with $P X R-, C A R$ - or control siRNA or no siRNA, and were seeded and incubated with paclitaxel for 0, 24, 48 or $72 \mathrm{~h}$ (B). TUNEL assay of apoptotic CaOV-3 cells transfected with $P X R$-, CAR- or control siRNA, or no siRNA and treated with paclitaxel for $36 \mathrm{~h}$ (C). Results show mean $\pm \mathrm{SD}$ of 5 independent experiments $\left({ }^{*} \mathrm{P}<0.01 ;{ }^{* *} \mathrm{P}<0.05\right.$ vs. control siRNA group, ${ }^{\prime} \mathrm{P}<0.01$ vs. CAR siRNA, $\$ \mathrm{P}<0.01$ vs. $P X R$ siRNA). 
enhanced cell growth inhibition in the presence of paclitaxel for 24,48 and $72 \mathrm{~h}$ compared with that of either knocked-down PXR or CAR alone (Fig. 7B). In the presence of paclitaxel for 48 and $72 \mathrm{~h}$, downregulating both PXR and CAR (i.e., in cells with both PXR and CAR siRNA transfections) significantly enhanced apoptosis compared with cells transfected with either PXR or CAR siRNA alone (Fig. 7C). Cell growth inhibition and apoptosis significantly differed between cells transfected with PXR siRNA and those transfected with CAR siRNA in the presence of paclitaxel (Fig. 7C).

\section{Discussion}

We investigated whether inhibition of the PXR-mediated pathway could affect cytotoxicity of the anticancer drugs, paclitaxel and/or cisplatin, in several ovarian cancer cell lines including CaOV-3, SKOV-3, and BG-1 cells, to examine the possible effect of PXR on augmentation of drug sensitivity and overcoming drug resistance. We observed that phthalate and pregnenolone had significant effects on the PXR-CYP3A4 pathway, whereas the PXR-MDR1 pathway was significantly increased in the presence of paclitaxel or cisplatin. We also observed that downregulating PXR strongly inhibited the augmentation of MDR1 expression and PXR-mediated transcription by PXR ligands, and significantly enhanced the cell growth inhibition and apoptosis in the presence of paclitaxel or cisplatin. In addition, we found that pregnenolone moderately and ketoconazole strongly suppressed the augmented MDR1 expression and PXR-mediated transactivation by paclitaxel or cisplatin, and enhanced cell-growth inhibition and apoptosis in the presence of paclitaxel or cisplatin. Cell growth inhibition and apoptosis were significantly enhanced in the cells transfected with $P X R$ siRNA compared with those transfected with $C A R$ siRNA in the presence of paclitaxel.

We previously demonstrated that PXR ligands enhance PXR-mediated transcription in a ligand- and promoter-dependent fashion, which in turn differentially regulate expression of individual PXR targets, such as CYP3A4 and MDR1, in endometrial cancer cells (29). We also observed that steroids and endocrine-disrupting chemicals enhanced CYP3A4 expression, whereas the anticancer agents, paclitaxel or cisplatin had positive effects on MDR1 expression in endometrial cancer cells (29). In this study, we observed that pregnenolone and phthalate mainly enhanced the CYP3A4 pathway, including mRNA expression and PXR-mediated transcription, but paclitaxel and cisplatin had stronger effects on the MDR1 pathway in ovarian cancer cells. This implies two different ligands and promoters, which differentially regulate expression of individual PXR targets, such as CYP3A4 and MDR1, in ovarian cancer cell lines.

MDR1 was originally identified because its overexpression in cultured cancer cells was associated with an acquired crossresistance to multiple anticancer drugs, and it has been shown to be an ATP-dependent efflux pump of hydrophobic anticancer drugs (7). Clinical paclitaxel resistance is often associated with MDR1 overexpression; in vitro paclitaxel resistance typically occurs with overexpression of the MDR1 gene (6). However, several clinical trials have attempted to alter P-glycoprotein activity and thus improve clinical outcomes using verapamil and dexamethasone; most of these studies showed no clear-cut success (9-11). An earlier report showed that siRNA targeted to MDRI could sensitize paclitaxel-resistant ovarian cancer cells in vitro (35), which suggests that siRNA treatment may present a new approach for treating MDR1-mediated drug resistance. Here, we showed that the mRNA levels of CYP3A4 and MDRI were strongly inhibited in cells transfected with $P X R$ siRNA, in the presence of PXR ligands, pregnenolone, phthalate, paclitaxel, and cisplatin, compared with cells treated with control siRNA and untransfected cells. In addition, we observed no PXR ligand that enhanced PXR-mediated transactivation through MDRI and CYP3A4 promoters in cells transfected with PXR siRNA. These data suggest that the PXR-CYP3A4 and PXR-MDR1 pathways are blocked by downregulated PXR in ovarian cancer cells. Next, we found that downregulating PXR expression significantly enhanced cell-growth inhibition and apoptosis in the presence of the anticancer agents, paclitaxel and cisplatin, which indicates that downregulating PXR in ovarian cancer might alter its response to anticancer agents.

Pregnenolone is an endogenous steroid hormone, known as a ligand for PXR $(36,37)$. It has a strong positive effect on the CYP3A4 pathway, compared with its effect on MDR1. The combination of cisplatin or paclitaxel with pregnenolone suppressed the MDR1 expression induced by anticancer agents in a dose-dependent manner. Because pregnenolone also suppressed PXR-mediated transcription through the $M D R 1$ promoter in the presence of paclitaxel or cisplatin, this suppression mediated by PXR, and the binding of pregnenolone to PXR might inhibit the PXR-mediated activation by paclitaxel and cisplatin. Ketoconazole, an antifungal drug, is reported to inhibit the PXR activation by binding to PXR (38) and to be a PXR antagonist, an inhibitor of both PXR-mediated drug metabolism and the MDR1 pathway in HepG2 cells (39). In this study, we showed that ketoconazole strongly inhibited augmentation of the MDR1 pathway by paclitaxel or cisplatin in dose-dependent manner. These data imply that pregnenolone partially suppressed the MDR1 pathway because pregnenolone weakly enhanced the MDR1 pathway through PXR. In contrast, ketoconazole strongly inhibited the MDR1 pathway because ketoconazole had no positive effect on PXR-mediated genes. The different inhibitory effects on the MDR1 pathway might have caused the different effects of pregnenolone and ketoconazole on cell growth inhibition and apoptosis in the presence of paclitaxel or cisplatin.

In this study, we observed PXR downregulation to suppress PXR-mediated transactivation through the MDR promoter, but it did not completely inhibit the paclitaxel or cisplatinaugmented MDR1 expression. Moreover, ketoconazole, a PXR antagonist, could not completely block the paclitaxel or cisplatin-enhanced MDR1 expression; and RNA interference of both $P X R$ and $C A R$ completely abolished MDR1 overexpression and enhanced cell growth inhibition and apoptosis in the presence of paclitaxel compared with interference of either PXR or CAR alone, which further indicates that both PXR- and CAR-mediated pathways affect drug resistance. We also observed that stronger effect of PXR downregulation on cell growth inhibition and apoptosis compared with $C A R$ downregulation in the presence of paclitaxel. PXR and CAR reportedly share several ligands including paclitaxel and cisplatin, and several target genes including $M D R 1$ $(21,22,29,30)$. Moreover, activation of phase I drug metabolism 
enzymes (such as CYP3A4) by PXR and/or CAR might affect the mechanism for drug resistance (40). Thus, the roles of xenobiotic receptors such as PXR and CAR in drug resistance for anticancer agents warrant further study.

In conclusion, inhibition of PXR-mediated pathways could augment sensitivity, or even overcome resistance, to anticancer agents in the treatment of ovarian cancer, suggesting a novel means for ovarian cancer patients, especially for those that have resistance to anticancer agents.

\section{Acknowledgements}

This study was supported in part by a research grant from the Ministry of Education, Culture, Sports, Science and Technology of Japan (no. 25462558).

\section{References}

1. Rubin SC, Randall TC, Armstrong KA, Chi DS and Hoskins WJ: Ten-year follow-up of ovarian cancer patients after second-look laparotomy with negative findings. Obstet Gynecol 93: 21-24, 1999.

2. Armstrong DK: Relapsed ovarian cancer: Challenges and management strategies for a chronic disease. Oncologist 7 (Suppl 5): 20-28, 2002

3. National Institute for Health and Care Excellence: Guidance on the use of paclitaxel in the treatment of ovarian cancer. http:/ www.nice.org.uk/guidance/ta55. Accessed January 22, 2003.

4. McGuire WP, Hoskins WJ, Brady MF, Kucera PR, Partridge EE, Look KY, Clarke-Pearson DL and Davidson M: Cyclophosphamide and cisplatin compared with paclitaxel and cisplatin in patients with stage III and stage IV ovarian cancer. N Engl J Med 334: 1-6, 1996.

5. Piccart MJ, Bertelsen K, James K, Cassidy J, Mangioni C, Simonsen E, Stuart G, Kaye S, Vergote I, Blom R, et al: Randomized intergroup trial of cisplatin-paclitaxel versus cisplatin-cyclophosphamide in women with advanced epithelial ovarian cancer: Three-year results. J Natl Cancer Inst 92 699-708, 2000.

6. Tsuruo T, Naito M, Tomida A, Fujita N, Mashima T, Sakamoto H and Haga N: Molecular targeting therapy of cancer: Drug resistance, apoptosis and survival signal. Cancer Sci 94: 15-21, 2003.

7. Ambudkar SV, Kimchi-Sarfaty C, Sauna ZE and Gottesman MM: P-glycoprotein: From genomics to mechanism. Oncogene 22 7468-7485, 2003.

8. Ling V: Multidrug resistance: Molecular mechanisms and clinical relevance. Cancer Chemother Pharmacol 40 (Suppl): S3-S8, 1997.

9. Dalton WS, Crowley JJ, Salmon SS, Grogan TM, Laufman LR, Weiss GR and Bonnet JD: A phase III randomized study of oral verapamil as a chemosensitizer to reverse drug resistance in patients with refractory myeloma. A Southwest Oncology Group study. Cancer 75: 815-820, 1995.

10. Sonneveld P, Suciu S, Weijermans P, Beksac M, Neuwirtova R, Solbu G, Lokhorst H, van der Lelie J, Dohner H, Gerhartz H, et al; European Organization for Research and Treatment of Cancer (EORTC); Leukaemia Cooperative Group (LCG); Dutch Haemato-Oncology Cooperative Study Group (HOVON): Cyclosporin A combined with vincristine, doxorubicin and dexamethasone (VAD) compared with VAD alone in patients with advanced refractory multiple myeloma: An EORTC-HOVON randomized phase III study (06914). Br J Haematol 115: 895-902, 2001.

11. Leonard GD, Fojo T and Bates SE: The role of ABC transporters in clinical practice. Oncologist 8: 411-424, 2003.

12. Kliewer SA, Moore JT, Wade L, Staudinger JL, Watson MA, Jones SA, McKee DD, Oliver BB, Willson TM, Zetterström RH, et al: An orphan nuclear receptor activated by pregnanes defines a novel steroid signaling pathway. Cell 92: 73-82, 1998.

13. Lehmann JM, McKee DD, Watson MA, Willson TM, Moore JT and Kliewer SA: The human orphan nuclear receptor PXR is activated by compounds that regulate CYP3A4 gene expression and cause drug interactions. J Clin Invest 102: 1016-1023, 1998.
14. Bertilsson G, Heidrich J, Svensson K, Asman M, Jendeberg L, Sydow-Bäckman M, Ohlsson R, Postlind H, Blomquist P and Berkenstam A: Identification of a human nuclear receptor defines a new signaling pathway for CYP3A induction. Proc Natl Acad Sci USA 95: 12208-12213, 1998.

15. Zhang H, LeCulyse E, Liu L, Hu M, Matoney L, Zhu W and Yan B: Rat pregnane X receptor: Molecular cloning, tissue distribution, and xenobiotic regulation. Arch Biochem Biophys 368: 14-22, 1999.

16. Blumberg B, Sabbagh W Jr, Juguilon H, Bolado J Jr, van Meter CM, Ong ES and Evans RM: SXR, a novel steroid and xenobiotic-sensing nuclear receptor. Genes Dev 12: 3195-3205, 1998.

17. Pascussi JM, Jounaidi Y, Drocourt L, Domergue J, Balabaud C, Maurel P and Vilarem MJ: Evidence for the presence of a functional pregnane $X$ receptor response element in the CYP3A7 promoter gene. Biochem Biophys Res Commun 260: 377-381, 1999.

18. Schuetz EG, BrimerC and Schuetz JD: Environmental xenobiotics and the antihormones cyproterone acetate and spironolactone use the nuclear hormone pregnenolone $\mathrm{X}$ receptor to activate the CYP3A23 hormone response element. Mol Pharmacol 54: 1113-1117, 1998

19. de Wildt SN, Kearns GL, Leeder JS and van den Anker JN: Cytochrome P450 3A: Ontogeny and drug disposition. Clin Pharmacokinet 37: 485-505, 1999.

20. Ketter TA, Flockhart DA, Post RM, Denicoff K, Pazzaglia PJ, Marangell LB, George MS and Callahan AM: The emerging role of cytochrome P450 3A in psychopharmacology. J Clin Psychopharmacol 15: 387-398, 1995.

21. Synold TW, Dussault I and Forman BM: The orphan nuclear receptor SXR coordinately regulates drug metabolism and efflux. Nat Med 7: 584-590, 2001.

22. Geick A, Eichelbaum M and Burk O: Nuclear receptor response elements mediate induction of intestinal MDR1 by rifampin. J Biol Chem 276: 14581-14587, 2001.

23. Kliewer SA, Goodwin B and Willson TM: The nuclear pregnane $\mathrm{X}$ receptor: A key regulator of xenobiotic metabolism. Endocr Rev 23: 687-702, 2002.

24. Hartley DP, Dai X, Yabut J, Chu X, Cheng O, Zhang T, He YD, Roberts C, Ulrich R, Evers R, et al: Identification of potential pharmacological and toxicological targets differentiating structural analogs by a combination of transcriptional profiling and promoter analysis in LS-180 and Caco-2 adenocarcinoma cell lines. Pharmacogenet Genomics 16: 579-599, 2006.

25. Guzelian J, Barwick JL, Hunter L, Phang TL, Quattrochi LC and Guzelian PS: Identification of genes controlled by the pregnane $\mathrm{X}$ receptor by microarray analysis of mRNAs from pregnenolone 16alpha-carbonitrile-treated rats. Toxicol Sci 94: 379-387, 2006.

26. Masuyama $\mathrm{H}$, Nakatsukasa $\mathrm{H}$, Takamoto $\mathrm{N}$ and Hiramatsu $\mathrm{Y}$ : Down-regulation of pregnane $\mathrm{X}$ receptor contributes to cell growth inhibition and apoptosis by anticancer agents in endometrial cancer cells. Mol Pharmacol 72: 1045-1053, 2007.

27. Gupta D, Venkatesh M, Wang H, Kim S, Sinz M, Goldberg GL, Whitney K, Longley $\mathrm{C}$ and Mani S: Expanding the roles for pregnane $\mathrm{X}$ receptor in cancer: Proliferation and drug resistance in ovarian cancer. Clin Cancer Res 14: 5332-5340, 2008.

28. Yue X, Akahira J, Utsunomiya H, Miki Y, Takahashi N, Niikura H, Ito K, Sasano H, Okamura K and Yaegashi N: Steroid and xenobiotic receptor (SXR) as a possible prognostic marker in epithelial ovarian cancer. Pathol Int 60: 400-406, 2010.

29. Masuyama H, Suwaki N, Tateishi Y, Nakatsukasa H, Segawa T and Hiramatsu Y: The pregnane X receptor regulates gene expression in a ligand- and promoter-selective fashion. Mol Endocrinol 19: 1170-1180, 2005.

30. Wang Y, Masuyama H, Nobumoto E, Zhang G and Hiramatsu Y: The inhibition of constitutive androstane receptor-mediated pathway enhances the effects of anticancer agents in ovarian cancer cells. Biochem Pharmacol 90: 356-366, 2014.

31. Xie W and Evans RM: Orphan nuclear receptors: The exotics of xenobiotics. J Biol Chem 276: 37739-37742, 2001.

32. Qatanani M and Moore DD: CAR, the continuously advancing receptor, in drug metabolism and disease. Curr Drug Metab 6: 329-339, 2005

33. Petru E, Sevin BU, Perras J, Boike G, Ramos R, Nguyen H and Averette HE: Comparative chemosensitivity profiles in four human ovarian carcinoma cell lines measuring ATP bioluminescence. Gynecol Oncol 38: 155-160, 1990. 
34. Smith JA, Ngo H, Martin MC and Wolf JK: An evaluation of cytotoxicity of the taxane and platinum agents combination treatment in a panel of human ovarian carcinoma cell lines. Gynecol Oncol 98: 141-145, 2005.

35. Duan Z, Brakora KA and Seiden MV: Inhibition of ABCB1 (MDR1) and ABCB4 (MDR3) expression by small interfering RNA and reversal of paclitaxel resistance in human ovarian cancer cells. Mol Cancer Ther 3: 833-838, 2004.

36. Masuyama H, Hiramatsu Y, Kunitomi M, Kudo T and MacDonald PN: Endocrine disrupting chemicals, phthalic acid and nonylphenol, activate Pregnane X receptor-mediated transcription. Mol Endocrinol 14: 421-428, 2000.

37. Masuyama H, Hiramatsu Y, Mizutani Y, Inoshita $\mathrm{H}$ and Kudo T: The expression of pregnane $\mathrm{X}$ receptor and its target gene, cytochrome P450 3A1, in perinatal mouse. Mol Cell Endocrinol 172: 47-56, 2001.
38. Wang H, Huang H, Li H, Teotico DG, Sinz M, Baker SD, Staudinger J, Kalpana G, Redinbo MR and Mani S: Activated pregnenolone $\mathrm{X}$-receptor is a target for ketoconazole and its analogs. Clin Cancer Res 13: 2488-2495, 2007.

39. Huang H, Wang H, Sinz M, Zoeckler M, Staudinger J, Redinbo MR, Teotico DG, Locker J, Kalpana GV and Mani S: Inhibition of drug metabolism by blocking the activation of nuclear receptors by ketoconazole. Oncogene 26: 258-268, 2007.

40. Chen Y, Tang Y, Guo C, Wang J, Boral D and Nie D: Nuclear receptors in the multidrug resistance through the regulation of drug-metabolizing enzymes and drug transporters. Biochem Pharmacol 83: 1112-1126, 2012. 